\title{
Metagenomics Study of the Microbes in Constructed Wetland System Treating Sewage
}

\author{
Boopathy Usharani
}

Centre for Environmental Studies, Anna University, Chennai

raniushab1@gmail.com

\author{
Keywords: Metagenomics; Next Generation Sequence; Bacteria; Fungi; Constructed Wetland \\ System.
}

\begin{abstract}
Constructed wetlands are eco-friendly, cost-effective technology involved in treatment of wastewaters. The goal of this study is focused on characterization of microbial community existing in a constructed wetland system planted with Cyperus alternifolius treating sewage contaminated with heavy metals. The characteristics of effluent met the standards of discharge for inland use and irrigation. Microbes in constructed wetland apparently play a pivotal role in the efficiency of system for removal of organics, nutrients, suspended solids and heavy metal. To expose the active players in the lime light, a representative soil sample from the reed bed was collected and characterized for microbial community analysis. Metagenomic studies of the bacterial and fungal flora were identified. Results revealed that the phylum Proteobacteria (38.27\%) and Ascomycota (77.47\%) dominated in the bacterial and fungal kingdom respectively. However, in the bacterial kingdom at species level major portion remain unclassified except Pseudomonas alcaligenes but in the fungal kingdom at species level only $3.1 \%$ remain unclassified. The role of bacteria in wastewater treatment is exemplified in previous reports but the role of fungi in the wastewater system needs exploration. However, the findings reveal that the identified microbes might have definitely played a vital role in wastewater treatment. The database available for the identification of bacterial species remain undiscovered for a major portion and requires upgradation. Next generation sequence being a highend technology in microbial ecology decodes the entire community in environmental samples but lack of database limits the identification. Implementation of improvements in the paucity of databases is essential.
\end{abstract}

\section{Introduction}

Constructed wetland system (CWS) is a clean green technology in the treatment of wastewater. The performance of CWS depends on biotic and abiotic factors [1]. Biotic participants involved in the treatment process are microbes and plants. A steady treatment in biological process needs exploration of microbial community residing in the system. Microbes in CWS play a crucial role in maintaining the ecological balance by degrading the environmental pollutants. Various studies were conducted to investigate the microbes responsible for degradation of pollutants in biological wastewater treatment. The phylum Proteobacteria dominates bioremediation sites [2]. Biological wastewater treatment involves biodegradation, oxidation and reduction of organic macromolecules and inorganic chemicals by residential microbes. Microbial biodegradation of pollutants existing in plant roots are well documented [3]. The microbial diversity in CWS is indispensable for the proper functioning of the system and is responsible for the improvement of water quality in a constructed wetland. Water, when exposed to diverse microbial species, favours nutrient removal in a short time and the composition of the microbes influence the water quality [4]. Microbes in the constructed wetland system plays a complex and vital role in the performance of CWS. Metagenomics paves way for the identification of microbes associated with plants in treatment process. The advent of NextGeneration Sequencing (NGS) technologies has made it possible to perform sequencing based metagenomic analysis. NGS aids in complete decoding of the entire community [5]. Healthy population of microbes in CWS is essential for the optimized removal of pollutants in the system. The population of the microbial community has a deserving part in the remediation techniques and 
should be brought forward in the limelight. The aim of the research is to unravel the taxonomy in CWS that played a vital role in the treatment process and this is the first report to identify the microbes in CWS treating sewage. Metagenomics is an advent and cutting edge technology in the field of microbial ecology which involves direct genetic analysis of genome in an environmental sample. The data obtained might provide an insight about the microbial ecosystem existing in CWS treating sewage. A strategy of enrichment of the identified populations can be executed for degradation of pollutants in the years to come.

\section{Theory}

The out comings of the research is based on the analysis of 16S rRNA of the bacterial and ITS of the fungal community in the rhizosphere soil sample tangled in treating sewage.

\section{Materials and Methods}

Composite soil sampling was done in CWS planted with CypeUrus alternifolius. Triplicate soil sampling was performed and homogenised soil sample was subjected to metagenomics study in Eurofins genomics lab, Bangalore.

\subsection{Metagenomic DNA isolation}

Metagenomic DNA isolation and qualitative/ quantitative analysis of the bacterial and fungal kingdom was carried out on 16S rDNA amplicon sequencing. Metagenomic DNA was isolated from the soil by using a commercially available kit (Nucleospin Soil), Macherey - Nagel, Germany. One $\mu 1$ of sample was loaded in Nano Drop for determining A260/280 ratio.

\subsection{Preparation of $2 \times 300$ MiSeq library}

The amplicon libraries were prepared using Nextera XT index kit (Illumina inc.USA) as per the $16 \mathrm{~S}$ metagenomic sequencing library preparation protocol. Primers for the amplification of $16 \mathrm{~S}$ rDNA gene specific for bacterial $\mathrm{V}_{3}-\mathrm{V}_{4}$ and ITS were designed and synthesised. Amplification of the 16S rDNA targeting bacteria and ITS were carried out. Three $\mu 1$ of PCR product was resolved on $1.2 \%$ agarose gel at $120 \mathrm{~V}$ for 60 minutes. Amplicons were synthesised using 2x KAPA HiFi HotStart ready mix (Kapa Biosystems) with forward and reverse primers presented in Table 3.1

Table 3.1 Primers used in metagenomic studies

\begin{tabular}{|l|l|}
\hline 16 S rRNA F & GCCTACGGGNGGCWGCAG \\
\hline 16 S rRNA R & ACTACHVGGGTATCTAATCC \\
\hline ITS- F & GCATCGATGAAGAACGCAGC \\
\hline ITS- R & TCCTCCGCTTATTGATATGC \\
\hline
\end{tabular}

The amplicons after a quality check with Illumina adaptors were amplified using i5 and i7 primers that add multiplexing index sequences as well as common adaptors required for cluster generation (P5 and P7) as per the standard Illumina protocol. The amplicon libraries were purified by AMPureXP beads and quantified using Qubit Fluorometer. The amplified libraries were analysed in 4200 Tape Station system (Agilent Technologies, USA) using D1000 Screen Tape.

\subsection{Cluster generation and sequencing}

After obtaining the mean peak size from Tape Station profile, libraries were loaded onto MiSeq at appropriate concentration $(10-20 \mathrm{pM})$ for cluster generation and sequencing. Paired- end sequencing allows the template fragments to be sequenced in both the forward and reverse directions on MiSeq. The kit reagents were used in binding of samples to complementary adapter oligos on paired-end flow cell. The adapters were designed to allow selective cleavage of the forward strands after re-synthesis of the reverse strand during sequencing. The copied reverse strand was then used to sequence from the opposite end of the fragment. 


\section{Result and Discussion}

Microbial metabolism plays a crucial role in the removal of pollutants from wastewater in biological system. As a preliminary step, amplification of 16S rDNA targeting bacteria and ITS were carried out after metagenomic DNA isolation. The PCR based amplicons generated on 1.2\% agarose gel is presented in Fig. 4.1

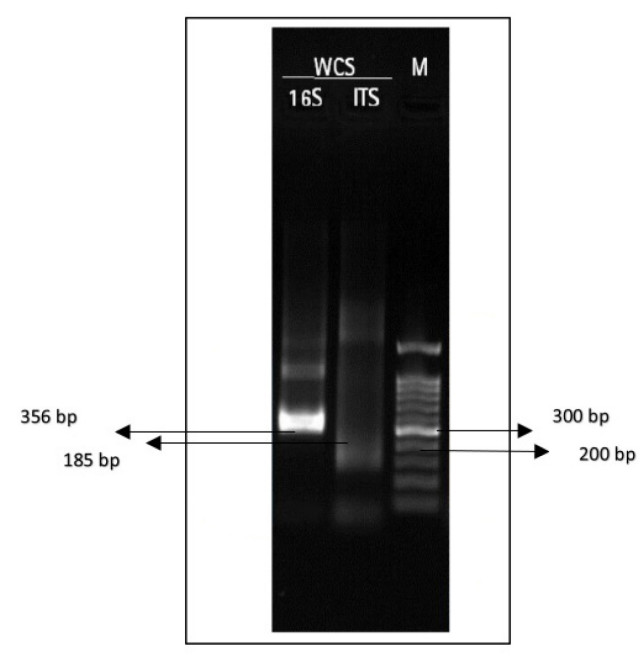

Figure 4.1. Amplified 16S rDNA and ITS genes on agarose gel

The amplified libraries were analysed in Tape Station for mean peak size of WCS 16S and mean peak size of ITS. The mean of the library fragment size distributions ranged between $588 \mathrm{bp}$ to 479bp for sample WCS-16S V3-V4 and ITS amplicon respectively. The libraries sequenced in the MiSeq platform revealed a total of 726, 838 reads with 356,415,976 total bases in high-quality fastq for WCS-16S. OTU ( Operational Taxonomic Unit) based sequence analysis approach was used with WCS (Whole Community Sequencing). The quantitative and qualitative results of the kingdom bacteria and fungi at each level: Phyla, class, order, family, genus and species are presented in individual pie charts.

\subsection{Bacterial community}

The relative abundance of bacterial phyla is presented in Fig. 4.2. Nineteen phyla were identified. It was inferred that the phyla Proteobacteria, Chlorflexi, Planctomycetes, Acidobacteria, Bacteroidetes and Actinobacteria contributed to $87.9 \%$ of the total sequences generated. Proteobacteria contributes a major fraction of the microbial biota.

In municipal wastewater treatment plants, Proteobacteria predominates (21-65\%) and the subdominates Chlorflexi, Acidobacteria and Bacteroidetes are majorly responsible for organic and nutrient removal in wastewater systems [6]. Proteobacteria, Acidobacteria, Actinobacteria, Bacteroidetes and Firmicutes accounted to $89.2 \%$ of the total OTUs in sites contaminated with $\mathrm{Zn}$ and $\mathrm{Cd}$ thus indicating that these phyla are resistant to heavy metal contamination. The most abundant taxa in heavy metal contaminated sites were Proteobacteria, Firmicutes and Actinobacteria. Proteobacteria (41.57\%), Bacteroidetes (11.78\%), Planctomycetes (9.36\%), Chlorflexi (7.6\%), Actinobactera (5.67\%) and Acidobacteria (3.27\%) were identified in the rhizosphere zone (Phragmites australis) of the CWS treating polluted rivers in Guangdong province located in south China [7]. Phylum Proteobacteria dominated in all the four constructed wetland systems planted with Typha latifolia and Salix atrocinerea in the treatment of municipal wastewater and swine wastewater [8]. 


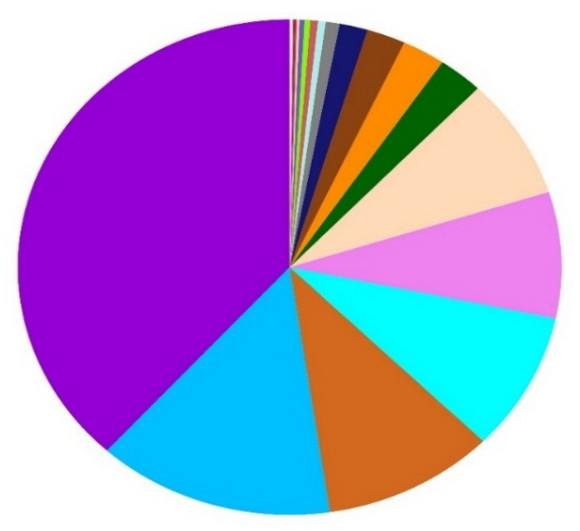

WCS16S Phylum legend

\begin{tabular}{|c|c|c|}
\hline Legends & Taxonomy & Abundance \\
\hline & k_Bacteria;p_Proteobacteria & $38.27 \%$ \\
\hline & k_Bacteria;p_Chloroflexi & $14.1 \%$ \\
\hline & k_Bacteria;p_Planctomycetes & $10.24 \%$ \\
\hline & k_Bacteria;p_Acidobacteria & $9.06 \%$ \\
\hline & k_Bacteria;p_Bacteroidetes & $8.24 \%$ \\
\hline & k_Bacteria;p_Actinobacteria & $7.93 \%$ \\
\hline & k_Bacteria;p_Firmicutes & $2.67 \%$ \\
\hline & k_Bacteria;p_Verrucomicrobia & $2.56 \%$ \\
\hline & k_Bacteria;p_Gemmatimonadetes & $2.31 \%$ \\
\hline & k_Bacteria;p_Nitrospirae & $1.67 \%$ \\
\hline & k_Bacteria;p_[Thermi] & $0.83 \%$ \\
\hline & k_Bacteria;p_TM7 & $0.46 \%$ \\
\hline & k_Bacteria;p_OD1 & $0.45 \%$ \\
\hline & k_Bacteria;p_Chlorobi & $0.34 \%$ \\
\hline k_Bacteria;p_TM6 & $0.3 \%$ \\
\hline & k_Bacteria;p_Cyanobacteria & $0.16 \%$ \\
\hline & k_Bacteria;p_Spirochaetes & $0.1 \%$ \\
\hline k_Bacteria;p_NC10 & $0.09 \%$ \\
\hline & k_Bacteria;p_NKB19 & $0.04 \%$ \\
\hline Others & $0.18 \%$ \\
\hline
\end{tabular}

Figure 4.2. Relative abundance of bacterial phyla

Previous reports indicate that the phyla Planctomycetes is responsible for the removal of dissolved organic matter and annamox process. Ammonia is utilized easier as it requires less energy for uptake. Acidobacteria and Verrucomicrobia are dominant in rhizoshere and are responsible for the plant-soil ecosystem. The first member of the phyla Gemmatimonadetes was identified as polyphosphate accumulating microorganism in activated sludge of the sewage treatment plant and might be responsible for the removal of phosphate from wastewater. Gemmatimonadetes has an exceptional quality of existing in both aerobic and anaerobic respiration as its metabolic pathways and enzyme involved are unique [9]. Nitrospirae is responsible for oxidation of nitrite in the system and $0.6 \%$ is reported in studies specifically conducted for the removal of nitrogen from swine wastewater via constructed wetlands [10]. Thermi is found to exist in summer and disappear during other seasons and has a positive correlation with temperature. TM7 is reported to be prevalent in acidophilic nitrifying sequencing batch reactors enabling the complete conversion of ammonium to nitrate. TM7 though not cultured yet, is observed in different environments including wastewater sludge. Phylum OD1 actively takes part in the efficient removal of ammonium [11]. Phyla Proteobacteria and OD1 increase remarkably in treating coliforms from wastewater using water hyacinth [12]. It might be responsible for the removal of coliforms in sewage.

Chlorobi is reported to be abundant in activated sludge and oxidises reduced sulphur compounds for carbondioxide fixation via the reverse of tricarboxylic acid (rTCA) cycle and accomplishes nitrogen fixation. TM6 is unculturable and exists in diverse habitats including the 
upflow anaerobic sludge blanket (UASB) reactor encoding genomes for oxidative stress tolerance. Cyanobacteria is abundant in eutrophicated water and is capable of uptaking nutrients, rapidly converting solar energy to carbon-based compounds. It converts nutrients from wastewater into biofuel. NC10 is an unculturable denitrifying methanotroph and an important member in biogeochemical cycle participating in conversion of nitrite to nitrogen. However the role of Spirochaetes and NKB19 in wastewater treatment are not yet reported in published literature.

The relative abundance \% of bacterial class identified from the library is presented in Fig. 4.3. A total of 19 bacterial class was identified. At class level, Alphaproteobacteria predominates which is a key representative of the microbial community in biological nitrogen fixation.

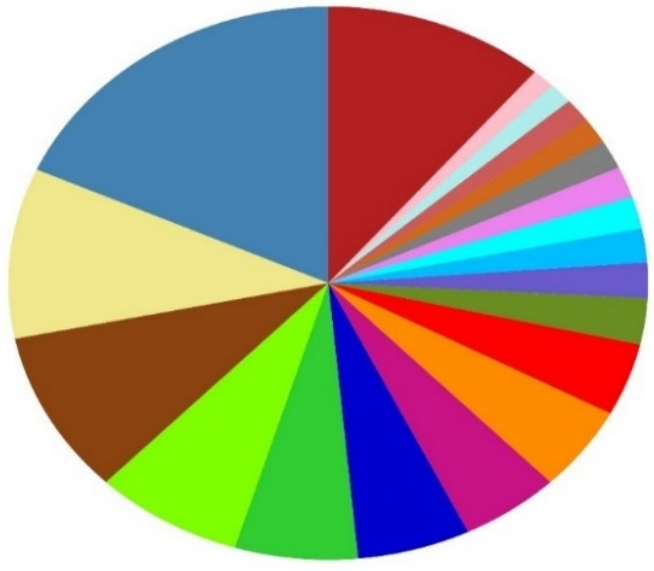

WCS16S Class legend

\begin{tabular}{|c|c|c|}
\hline Legends & Taxonomy & Abundance \\
\hline & k_Bacteria;p_Proteobacteria;c_Alphaproteobacteria & $18.27 \%$ \\
\hline & k_Bacteria;p_Proteobacteria;c_Gammaproteobacteria & $9.96 \%$ \\
\hline & k_Bacteria;p_Planctomycetes;c_Planctomycetia & $9.55 \%$ \\
\hline & k_Bacteria;p_Chloroflexi;c_Anaerolineae & $7.5 \%$ \\
\hline & k_Bacteria;p_Bacteroidetes;c_Cytophagia & $6.22 \%$ \\
\hline & k_Bacteria;p_Acidobacteria;c_Solibacteres & $5.71 \%$ \\
\hline & k_Bacteria;p_Proteobacteria;c_Deltaproteobacteria & $5.02 \%$ \\
\hline & k_Bacteria;p_Actinobacteria;c_Actinobacteria & $4.97 \%$ \\
\hline & k_Bacteria;p_Proteobacteria;c_Betaproteobacteria & $4.24 \%$ \\
\hline & k_Bacteria;p_Chloroflexi;c_Ellin6529 & $2.7 \%$ \\
\hline & k_Bacteria;P_Chloroflexi;c_Gitt-GS-136 & $2.07 \%$ \\
\hline & k_Bacteria;p_Actinobacteria;c_Acidimicrobiia & $1.97 \%$ \\
\hline & k_Bacteria;p_Gemmatimonadetes;c_Gemmatimonadetes & $1.88 \%$ \\
\hline & k_Bacteria;p_Acidobacteria;c_Acidobacteria-6 & $1.81 \%$ \\
\hline & k_Bacteria;p_Nitrospirae;c_Nitrospira & $1.67 \%$ \\
\hline & k_Bacteria;p_Firmicutes;c_Bacilli & $1.5 \%$ \\
\hline & k_Bacteria;p_Verrucomicrobia;c_[Pedosphaerae] & $1.41 \%$ \\
\hline & k_Bacteria;p_Acidobacteria;c_[Chloracidobacteria] & $1.21 \%$ \\
\hline & k_Bacteria;p_Bacteroidetes;c_[Saprospirae] & $1.17 \%$ \\
\hline & Others & $11.18 \%$ \\
\hline
\end{tabular}

Figure 4.3. Relative abundance of bacterial class

Alphaproteobacteria and Gammaproteobacteria might be the reason for the effective removal of organic pollutants and heavy metals. Planctomycetia plays a pivotal role in the biological nitrogen cycle producing approximately half of the $\mathrm{N}_{2}$ gas in the atmosphere. Planctomycetia might be responsible for the removal of ammonium and nitrite. Anaerolinae is earlier reported to be present in the activated sludge of SBR; likely participating in the removal of phosphorus in wastewater. Cytophagia performs hydrolysis of cellulose and lignocellulose releasing products which are utilised as carbon source. 
Alphaproteobacteria possess trancriptional regulatory network involved in nitrogen fixation was studied in depth [13] and might have played a key role in nitrogen removal. It is one of the major bacterial community (24\%) in soil amended with sewage sludge contaminated with heavy metal in varied concentration. Alphaproteobacteria, Gammaproteobacteria and Firmicutes have specific functional genes for sulphate reduction and heavy metal homeostasis [14]. The reduction of sulphate might have helped in the conversion of metal into inert metal sulphides.

About $11.6 \%$ of Cytophagia is reported in anaerobic digestors, $14.8 \%$ in hospital wastewater and $1.4 \%$ in domestic wastewater. The abundance of Betaproteobacteria,. Deltaproteobacteria, Cytophagia and Flavobacteria are majorly responsible for organic and nutrient removal in WWTP [15]. Solibacteres $(5.71 \%)$ is reported to be present in wetlands of Kolkata. However, its presence or role in WWTP is not yet reported.

Deltaproteobacteria reduces sulphate and is capable of surviving in eutrophic lakes and is effective in removal of nutrients. Dominance of Actinobacteria is reported in wastewater treatment plant, aeration basins and seawater processing wastewater treatment plant. Class Betaproteobacteria is responsible for nutrient and organic removal [15, 6]. Ellin6529 is reported to be present in surface sediments of Helgoland mud area, North sea adapted to fresh organic matter. Gitt GS136 is identified in the sandy soil of apple orchard positively correlated to manure and unexplored cave of Bat Guano. However, its presence and role in WWTP is not explored yet.

Gemmatimonadetes is abundant in activated sludge of WWTP and might participate in phosphate removal. The subdivision Acidobacteria 6 is reported earlier in agricultural soil and marine environment and possess conserved genome of evolutionary and ecological significance but does not have valid taxonomic names [16]. Nitrospira is responsible for nitrification in natural and engineered system and removal of nitrogen in biological wastewater treatment process [6]. Class Pedosphaerae in phyla Verrucomicrobia strongly influences the $\mathrm{pH}$ and $\mathrm{C}: \mathrm{N}$ ratio of the soil. Chloracidobacteria in membrane aerated biofilm reactor had a positive correlation with removal rate of COD and ammoniacal nitrogen [17].

The relative abundance of bacterial order identified from the library is presented in Fig. 4.4. A total of 19 bacterial orders were identified. At the order level; Cytophagales predominates. Solibacterales is reported to have a positive correlation with rice cropping in paddy fields. Sphingomonadales are phototropic and chemoorganotrophic, active under oxic conditions and survive in nutrient lacking systems

Cytophagales dominance favours nutrient and trace organic contaminant removal. Sphingomonadales produce extracellular polymeric substances that support biomass production [14]. Actinomycetales is one among the 10 dominant bacterial orders in WWTP. It is identified to own the genes for nitrogen metabolism in WWTP. Rhodospirillales and Sphingomonadales represent the biofilm communities and retains antibiotic resistant genes [17]. Rhizobiales release extracellular polymeric substances that support biomass production [14] and possess genes for nitrogen metabolism. It degrades complex organic compounds and prevents bio-fouling. 


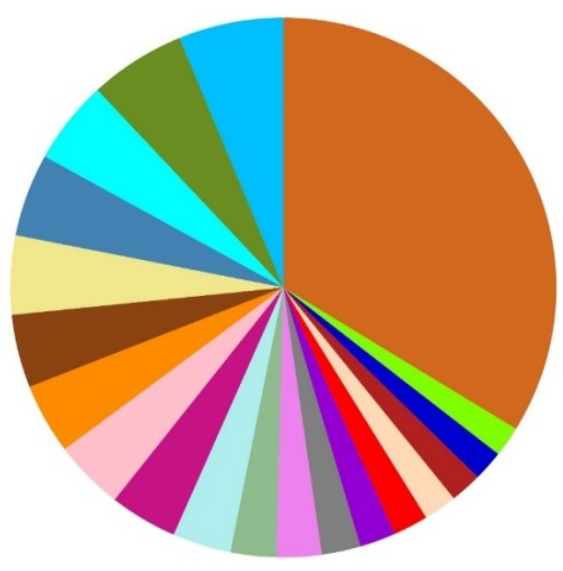

WCS16S Order legend

\begin{tabular}{|c|c|c|}
\hline Legends & Taxonomy & Abundance \\
\hline & k_Bacteria;p_Bacteroidetes;c_Cytophagia;0_Cytophagales & $6.22 \%$ \\
\hline & k_Bacteria;p_Acidobacteria;c_Solibacteres;0_Solibacterales & $5.71 \%$ \\
\hline & k_Bacteria;p_Proteobacteria;c_Alphaproteobacteria;0_Sphingomonadales & $5.0 \%$ \\
\hline & k_Bacteria;p_Actinobacteria;c_Actinobacteria;0_Actinomycetales & $4.96 \%$ \\
\hline & k_Bacteria;p_Proteobacteria;c_Alphaproteobacteria;0_Rhodospirillales & $4.73 \%$ \\
\hline & k_Bacteria;p_Proteobacteria;c_Alphaproteobacteria;0_Rhizobiales & $4.38 \%$ \\
\hline & k_Bacteria;p_Proteobacteria;c_Gammaproteobacteria;0_Xanthomonadales & $4.22 \%$ \\
\hline & k_Bacteria;p_Planctomycetes;c_Planctomycetia;0_Gemmatales & $4.18 \%$ \\
\hline & k_Bacteria;p_Chloroflexi;c_Anaerolineae;0_Anaerolineales & $4.02 \%$ \\
\hline & k_Bacteria;p_Planctomycetes;c_Planctomycetia;0_Planctomycetales & $3.47 \%$ \\
\hline & k_Bacteria;p_Chloroflexi;c_Ellin6529;0_Unclassified & $2.7 \%$ \\
\hline & k_Bacteria;p_Proteobacteria;c_Deltaproteobacteria;0_Myxococcales & $2.66 \%$ \\
\hline & k_Bacteria;p_Proteobacteria;c_Gammaproteobacteria;0_Pseudomonadales & $2.31 \%$ \\
\hline & k_Bacteria;p_Proteobacteria;c_Alphaproteobacteria;0_Rhodobacterales & $2.13 \%$ \\
\hline & k_Bacteria;P_Chloroflexi;c_Gitt-GS-136;0_Unclassified & $2.07 \%$ \\
\hline & k_Bacteria;p_Actinobacteria;c_Acidimicrobiia;0_Acidimicrobiales & $1.97 \%$ \\
\hline & k_Bacteria;p_Planctomycetes;c_Planctomycetia;0_Pirellulales & $1.88 \%$ \\
\hline & k_Bacteria;p_Gemmatimonadetes;c_Gemmatimonadetes;0_Unclassified & $1.81 \%$ \\
\hline & k_Bacteria;p_Acidobacteria;c_Acidobacteria-6;0_iii1-15 & $1.81 \%$ \\
\hline & Others & $33.77 \%$ \\
\hline
\end{tabular}

Figure 4.4. Relative abundance of bacterial order

Xanthomonadales is reported as a biofilm constructor in PVC membranes in MBR and common microbiota in all activated sludge [6]. Xanthomonadales is active under oxic conditions and survive in nutrient lacking systems. Anaerolineales represented 6.73\% in anaerobic digestors of WWTP and capable of conducting chemoorganotrophic decomposition [15]. Anaerolineales, Planctomycetales and Myxococcales are common members of WWTP [6].

Pseudomonadales thrive in oligotrophic environment and active under oxic conditions and survive in nutrient lacking systems. It is dominant in MBBR systems. Pseudomonadales (36.5\%) in oil field producing WWTP are capable of degrading petroleum products. Rhodobacterales is abundant in MBBR systems, highly stable in WWTP owing antibiotic resistant genes [18]. Acidomicrobiales are oligotrophs accounting 3.9\% in enroflaxin treatment systems. Pirellulales is abundant in MBBR biofilm. However, the order iii 1-15 is not repoted previously in cited literatures.

The relative abundance \% of bacterial family identified from the library is presented in Fig. 4.5. Total of 13 families were identified while 6 remain unclassified. Among the identified families, Cytophagaceae dominates. Sphingomonadaceae family members might have played a key role in remediation of heavy metals in sewage. Anaerolinaceae is involved in granular formation in activated 
sludge responsible for methanogenesis. Rhodospirillaceae is capable of assimilating organic compounds under anaerobic conditions and chemotrophic growth under aerobic conditions. Members of this family are entangled in nitrogen fixation and nitrogenase activity. Plancomycetes in Planctomycetaceae and Gemmata in Gemmataceae are key members in annamox reactors, specially designed for releasing fixed nitrogen back into atmosphere and $\mathrm{NH}_{4} \mathrm{~N}$ removal from wastewater.

Cytophagaceae exists in diverse habitats inclusive of activated sludge and wastewater exerting effective reduction of overall pathogens in wastewater. It might be the cause for reduction of coliforms in CWS. Members of Sphingomonadaceae family are dominant in rhizosphere containing root exudates, fruiting bodies of ectomycorrhizal fungi, microbial mats involved in self-purification of mine waters contaminated with heavy metals. Sphingomonadaceae isolated from different places acts as reservoirs of antibiotic resistance [19].

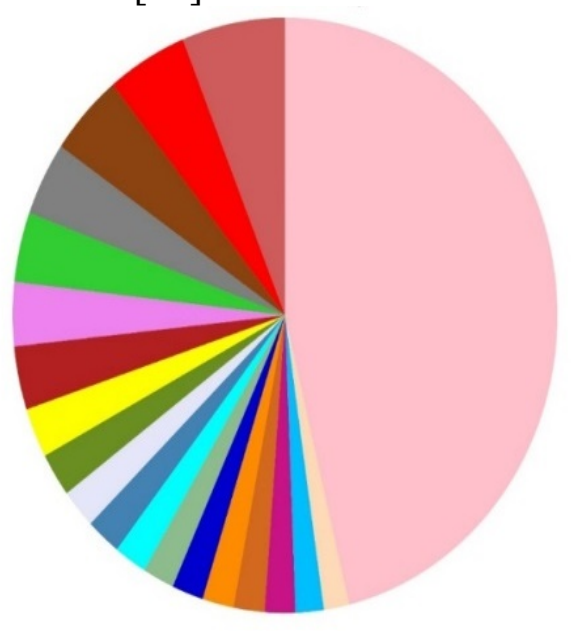

WCS16S Family legend

\begin{tabular}{|c|c|c|}
\hline Legends & Taxonomy & Abundance \\
\hline & k_Bacteria;p_Bacteroidetes;c_Cytophagia;0_Cytophagales;f_Cytophagaceae & $6.13 \%$ \\
\hline & k_Bacteria;P_Proteobacteria;c_Alphaproteobacteria;0_Sphingomonadales;f_Sphingomonadaceae & $4.77 \%$ \\
\hline & k_Bacteria;p_Acidobacteria;c_Solibacteres;0_Solibacterales;f_Unclassified & $4.5 \%$ \\
\hline & k_Bacteria;p_Chloroflexi;c_Anaerolineae;0_Anaerolineales;f_Anaerolinaceae & $4.02 \%$ \\
\hline & k_Bacteria;p_Proteobacteria;c_Alphaproteobacteria;0_Rhodospirillales;f_Rhodospirillaceae & $3.79 \%$ \\
\hline & k_Bacteria;p_Planctomycetes;c_Planctomycetia;0_Planctomycetales;f_Planctomycetaceae & $3.47 \%$ \\
\hline & k_Bacteria;p_Planctomycetes;c_Planctomycetia;0_Gemmatales;f_Gemmataceae & $3.45 \%$ \\
\hline & k_Bacteria;p_Chloroflexi;c_Ellin6529;0_Unclassified;f_Unclassified & $2.7 \%$ \\
\hline & k_Bacteria;p_Proteobacteria;c_Gammaproteobacteria;0_Xanthomonadales;f_Xanthomonadaceae & $2.38 \%$ \\
\hline & k_Bacteria;p_Proteobacteria;c_Deltaproteobacteria;0_Myxococcales;f_Unclassified & $2.14 \%$ \\
\hline & k_Bacteria;p_Chloroflexi;c_Gitt-GS-136;0_Unclassified;f_Unclassified & $2.07 \%$ \\
\hline & k_Bacteria;p_Proteobacteria;c_Alphaproteobacteria;0_Rhizobiales;f_Hyphomicrobiaceae & $1.95 \%$ \\
\hline & k_Bacteria;P_Planctomycetes;c_Planctomycetia;0_Pirellulales;f_Pirellulaceae & $1.88 \%$ \\
\hline & k_Bacteria;p_Proteobacteria;c_Gammaproteobacteria;0_Pseudomonadales;f_Pseudomonadaceae & $1.87 \%$ \\
\hline & k_Bacteria;p_Proteobacteria;c_Alphaproteobacteria;0_Rhodobacterales;f_Rhodobacteraceae & $1.86 \%$ \\
\hline & k_Bacteria;p_Proteobacteria;c_Gammaproteobacteria;0_Xanthomonadales;f_Sinobacteraceae & $1.84 \%$ \\
\hline & k_Bacteria;p_Gemmatimonadetes;c_Gemmatimonadetes;0_Unclassified;f_Unclassified & $1.81 \%$ \\
\hline & k_Bacteria;p_Nitrospirae;c_Nitrospira;0_Nitrospirales;f_Nitrospiraceae & $1.67 \%$ \\
\hline & k_Bacteria;p_Proteobacteria;c_Gammaproteobacteria;0_34P16;f_Unclassified & $1.51 \%$ \\
\hline & Others & $46.22 \%$ \\
\hline
\end{tabular}

Figure 4.5. Relative abundance of bacterial family 
Anaerolinaceae are involved in granular formation in activated sludge responsible for methanogenesis. Rhodospirillaceae is capable of assimilating organic compounds under anaerobic conditions and chemotrophic growth under aerobic conditions. Members of this family are entangled in nitrogen fixation and nitrogenase activity. Plancomycetes in Planctomycetacea and Gemmata in Gemmataceae are key members in annamox reactors, specially designed for releasing fixed nitrogen back into atmosphere and $\mathrm{NH}_{4} \mathrm{~N}$ removal from wastewater. Xanthomonadaceae is reported to exist in WWTP and activated sludge capable of metabolising hydrocarbon and nutrient removal in aerobic granular sludge wastewater treatment systems. They produce extracellular polymeric substances that support biomass production [15]. Hyphomicrobiaceae positively correlates with $\mathrm{NH}_{4} \mathrm{~N}$ removal [20] and removal of micropollutants in ecological WWTP. Pirellulaceae acts as soil indicator microbe, strongly correlated with soil $\mathrm{pH}$ and increases at neutral $\mathrm{pH}$. Members of Pseudomonadaceae family actively participate in the removal of heavy metals: $\mathrm{Fe}, \mathrm{Pb}, \mathrm{Zn}, \mathrm{Cu}, \mathrm{Ni}, \mathrm{Cr}$ and nitrate. The role of Rhodobacteraceae and Sinobacteraceae is yet to be explored. Nitrospiraceae plays a vital role in sulphate reduction in WWTP [21].

At genus level only 6 bacterial sequences were identified while others remain unclassified. The relative abundance $\%$ of bacterial genera identified from the library is presented in Fig. 4.6. Some of the species are capable of metabolising diverse chemical pollutants including xenobiotics. Though metagenomic studies reveal novel microbes, large fraction still remains unclassified in Next Generation Sequencing (NGS) technology enabling the detection of unculturable and unclassified microbes.

The genera Planctomyces and Gemmata were earlier reported to be in annamox reactor. The Genus Nitrospira plays a vital role in the removal of organic matter and nitrogen from the system. The detection of these three genera revealed that it might have played a crucial role in the removal of nitrogen from wastewater. Anaerolinea is a filamentous organism reported to be present in municipal and textile WWTP, with the ability to generate energy by fermentation contributing denitrification process [22]. 


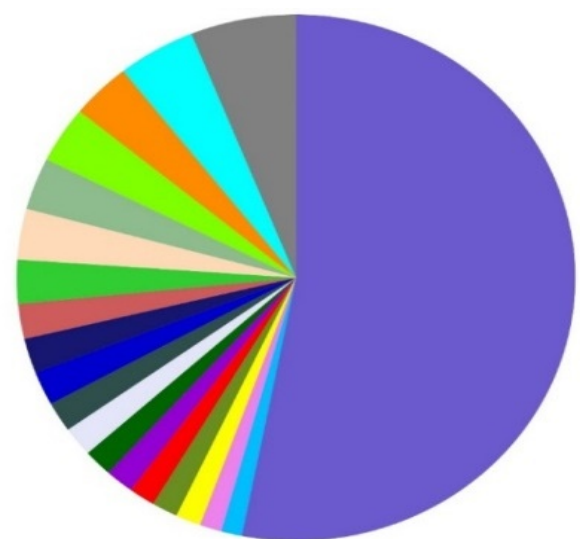

WCS16S Genus legend

\begin{tabular}{|c|c|c|}
\hline Legends & Taxonomy & Abundance \\
\hline & k_Bacteria;p_Bacteroidetes;c_Cytophagia;o_Cytophagales;f_Cytophagaceae;'g_Unclassified & $6.12 \%$ \\
\hline & k_Bacteria;p_Acidobacteria;c_Solibacteres;0_Solibacterales;f_Unclassified;g_Unclassified & $4.5 \%$ \\
\hline & k_Bacteria;p_Proteobacteria;__Alphaproteobacteria;0_Rhodospirillales;f_Rhodospirillaceae;'g_Unclassified & $3.49 \%$ \\
\hline & k_Bacteria;p_Planctomycetes;__Planctomycetia;o_Planctomycetales;f_Planctomycetaceae;g_Planctomyces & $3.47 \%$ \\
\hline & k_Bacteria;p_Planctomycetes;c_Planctomycetia;0_Gemmatales;f_Gemmataceae;g_Gemmata & $3.2 \%$ \\
\hline & k_Bacteria;;_Chloroflexi;c_Anaerolineae;0_Anaerolineales;f_Anaerolinaceae;g_Anaerolinea & $3.12 \%$ \\
\hline & k_Bacteria;p_Chloroflexi;c_Ellin6529;0_Unclassified;f_Unclassified;g_Unclassified & $2.7 \%$ \\
\hline & k_Bacteria;p_Proteobacteria;__Alphaproteobacteria;0_Sphingomonadales;__Sphingomonadaceae;g_Kaistobacter & $2.14 \%$ \\
\hline & k_Bacteria;p_Proteobacteria;__Deltaproteobacteria;0_Myxococcales;f_Unclassified;g_Unclassified & $2.14 \%$ \\
\hline & k_Bacteria;p_Chloroflexi;__Gitt-GS-136;0_Unclassified;__Unclassified;g_Unclassified & $2.07 \%$ \\
\hline & k_Bacteria;p_Proteobacteria;c_Gammaproteobacteria;0_Pseudomonadales;f_Pseudomonadaceae;g_Pseudomonas & $1.85 \%$ \\
\hline & k_Bacteria;p_Gemmatimonadetes;c_Gemmatimonadetes;0_Unclassified;__Unclassified;g_Unclassified & $1.81 \%$ \\
\hline & k_Bacteria;p_Proteobacteria;c_Gammaproteobacteria;0_Xanthomonadales;f_Sinobacteraceae;g_Unclassified & $1.67 \%$ \\
\hline & k_Bacteria;p_Nitrospirae;_c_Nitrospira;0_Nitrospirales,f_Nitrospiraceae;g_Nitrospira & $1.66 \%$ \\
\hline & k_Bacteria;i_Proteobacteria;__Alphaproteobacteria;0_Sphingomonadales;f_Sphingomonadaceae;'g_Unclassified & $1.51 \%$ \\
\hline & k_Bacteria;p_Proteobacteria;c_Gammaproteobacteria;0_34P16;f_Unclassified;g_Unclassified & $1.51 \%$ \\
\hline & k_Bacteria;p_Proteobacteria;c_Deltaproteobacteria;0_Syntrophobacterales;f_Syntrophobacteraceae;g_Unclassified & $1.46 \%$ \\
\hline & k_Bacteria;P_Planctomycetes;c_Planctomycetia;0_Pirellulales;f_Pirellulaceae;g_Unclassified & $1.27 \%$ \\
\hline & k_Bacteria;p_Proteobacteria;c_Betaproteobacteria;0_Burkholderiales;f_Comamonadaceae;g_Unclassified & $1.2 \%$ \\
\hline & Others & $53.12 \%$ \\
\hline
\end{tabular}

Figure 4.6. Relative abundance of bacterial genera

Though the function of Kaistobacter is not reported earlier, its presence is detected in methaneenriched soil and paddy fields attracting scientists to explore its role in bioremediation [23]. Though the genus Pseudomonas, isolated from both fresh water and wastewater are antibiotic resistant, its enzymic activity encounter organic sulphur removal preventing malodorous compound release and denitrification [24]. Pseudomonas might have contributed for the devoid of malodorous odour in the effluent.

The relative abundance $\%$ of bacterial species identified from the library is presented in Fig. 4.7. At species level, the metagenomic study majorly revealed unclassified species due to lack of available tools and paucity of reference genomes in public databases. Despite the use of NCBI database, unclassified bacteria accounted for $69.46 \%$ of the total bacteria. Unclassified species belongs to the phylum Proteobacteria, Planctomycetes, Nitrospirae, Gemmatimonadetes, Chlorflexi, Acidobacteria and Bacteroidetes. All the above-represented phyla are reported to be dominant and actively participate in the removal of organics, nutrients and heavy metals.

The only identified species was Pseudomonas alcaligenes. Alphaproteobacteria and the unclassified members of the Proteobacteria constitites $16.41 \%$ of the total members at species level in the present study. Pseudomonas alcaligenes occurrs very rare and is involved in bioremediation of polluted sites [25]. Microorganisms such as alcaligenes sp induces beneficial response to plants when present in soil. A positive correlation existed between Alphaproteobacteria, unclassified members of 
the Proteobacteria and the heavy metal contaminated site especially mercury. Most of the species of the Comamonadaceae have flagella. The unclassified members of the Comamonadaceae are involved in the biological nutrient removal process, especially nitrogen from wastewater when the oxygen supply is limited [26].

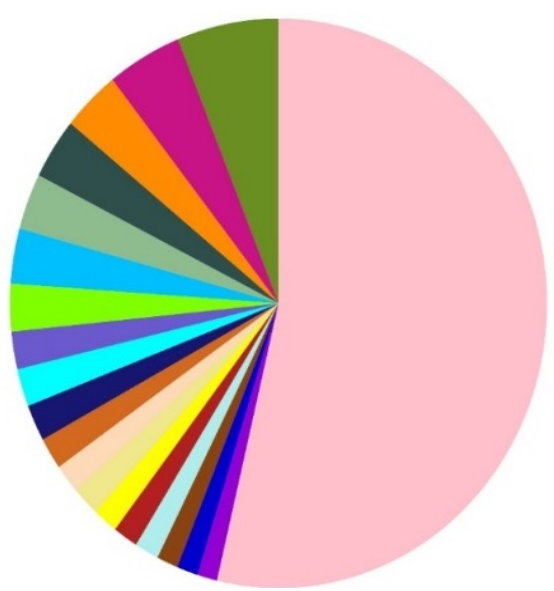

WCS16S Species legend

\begin{tabular}{|c|c|c|}
\hline Legends & Taxonomy & Abundance \\
\hline & k_Bacteria;p_Bacteroidetes;__Cytophagia;0_Cytophagales;__Cytophagaceae;'g_Unclassified;s_Unclassified & $6.12 \%$ \\
\hline & k_Bacteria;p_Acidobacteria;'c_Solibacteres;;0_Solibacterales;;_Unclassified;'g_Unclassified;'s_Unclassified & $4.5 \%$ \\
\hline & k_Bacteria;p_Proteobacteria;c_Alphaproteobacteria;0_Rhodospirillales;__Rhodospirillaceae;'g_Unclassified;s_Unclassified & $3.49 \%$ \\
\hline & k_Bacteria;p_Planctomycetes;___Planctomycetia;0_Planctomycetales;__Planctomycetaceae;g_Planctomyces;s_Unclassified & $3.47 \%$ \\
\hline & k_Bacteria;p_Planctomycetes;__Planctomycetia;0_Gemmatales;__Gemmataceae;g_Gemmata;s_Unclassified & $3.19 \%$ \\
\hline & k_Bacteria;p_Chloroflexi;c_Anaerolineae;0_Anaerolineales;_f_Anaerolinaceae;'g_Anaerolinea;s_Unclassified & $3.12 \%$ \\
\hline & k_Bacteria;p_Chloroflexi;c_Ellin6529;0_Unclassified;;_Unclassified;g_Unclassified;s_Unclassified & $2.7 \%$ \\
\hline & k_Bacteria;p_Proteobacteria;c_Alphaproteobacteria;0_Sphingomonadales;__Sphingomonadaceae;g_Kaistobacter;s_Unclassified & $2.14 \%$ \\
\hline & k_Bacteria;p_Proteobacteria;'c_Deltaproteobacteria;0_Myxococcales;__Unclassified;g_Unclassfified;s_Unclassified & $2.14 \%$ \\
\hline & k_Bacteria;p_Chloroflexi;c__iitt-GS-136;0_Unclassified;f_Unclassified;g_Unclassified;s_Unclassified & $2.07 \%$ \\
\hline & k_Bacteria;p_Gemmatimonadetes;_c_Gemmatimonadetes;'0_Unclassified;f_Unclassified;g_Unclassified;s_Unclassified & $1.81 \%$ \\
\hline & k_Bacteria;i_Proteobacteria;___Gammaproteobacteria;0_Xanthomonadales;,_Sinobacteraceae;'G_Unclassified;s_Unclassified & $1.67 \%$ \\
\hline & k_Bacteria;i_Nitrospirae;c_Nitrospira;0_Nitrospirales;f_Nitrospiraceae;g_Nitrospira;s_Unclassified & $1.66 \%$ \\
\hline & k_Bacteria;p_Proteobacteria;c_Alphaproteobacteria;0_Sphingomonadales;;_Sphingomonadaceae;'g_Unclassified;'s_Unclassified & $1.51 \%$ \\
\hline & k_Bacteria;p_Proteobacteria;c_Gammaproteobacteria;0_34P16;__Unclassified;g_Unclassified;s_Unclassified & $1.51 \%$ \\
\hline & k_Bacteria;p_Proteobacteria;c_Deltaproteobacteria;0_Syntrophobacterales;'_Syntrophobacteraceae'g_Unclassified;s_Unclassified & $1.46 \%$ \\
\hline & k_Bacteria'p_Proteobacteria;c_Gammaproteobacteria;0_Pseudomonadales;;_Pseudomonadaceae;g_Pseudomonas;s_alcaligenes & $1.29 \%$ \\
\hline & k_Bacteria;i_Planctomycetes;'__Planctomycetia;,__Pirellulales;;_Pirellulaceae;g_Unclassified;;__Unclassified & $1.27 \%$ \\
\hline & k_Bacteria;p_Proteobacteria;'__Betaproteobacteria;;_B_Burkholderiales;__Comamonadaceae;'__Unclassified;s_Unclassified & $1.2 \%$ \\
\hline & Others & $53.69 \%$ \\
\hline
\end{tabular}

Figure 4.7. Relative abundance of bacterial species

Diversity and rarefaction measure of observed OTUs of the bacterial genome was calculated using $\alpha$ diversity metrics is presented in Fig. 4.8. 


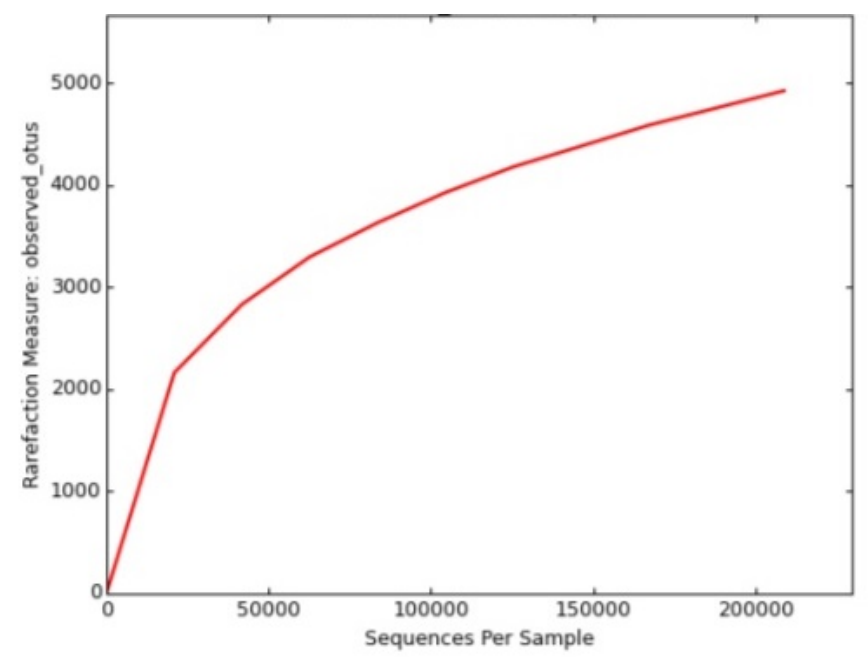

Figure 4.8. Rarefaction measure of observed OTUs in bacterial genome

Total of 3373 OTUs were observed with a shanon alpha diversity of 9.156. The species richness can be calculated from rarefaction curve. Steep slope on the left indicates that a large fraction of the species remains to be discovered. The vertical axis displays the diversity of community and horizontal axis displays the number of sequences considered in the diversity calculation. The whole community sequencing of bacteria is presented in Fig. 4.9 using Krona software. It revealed $0.06 \%$ of Archaea.

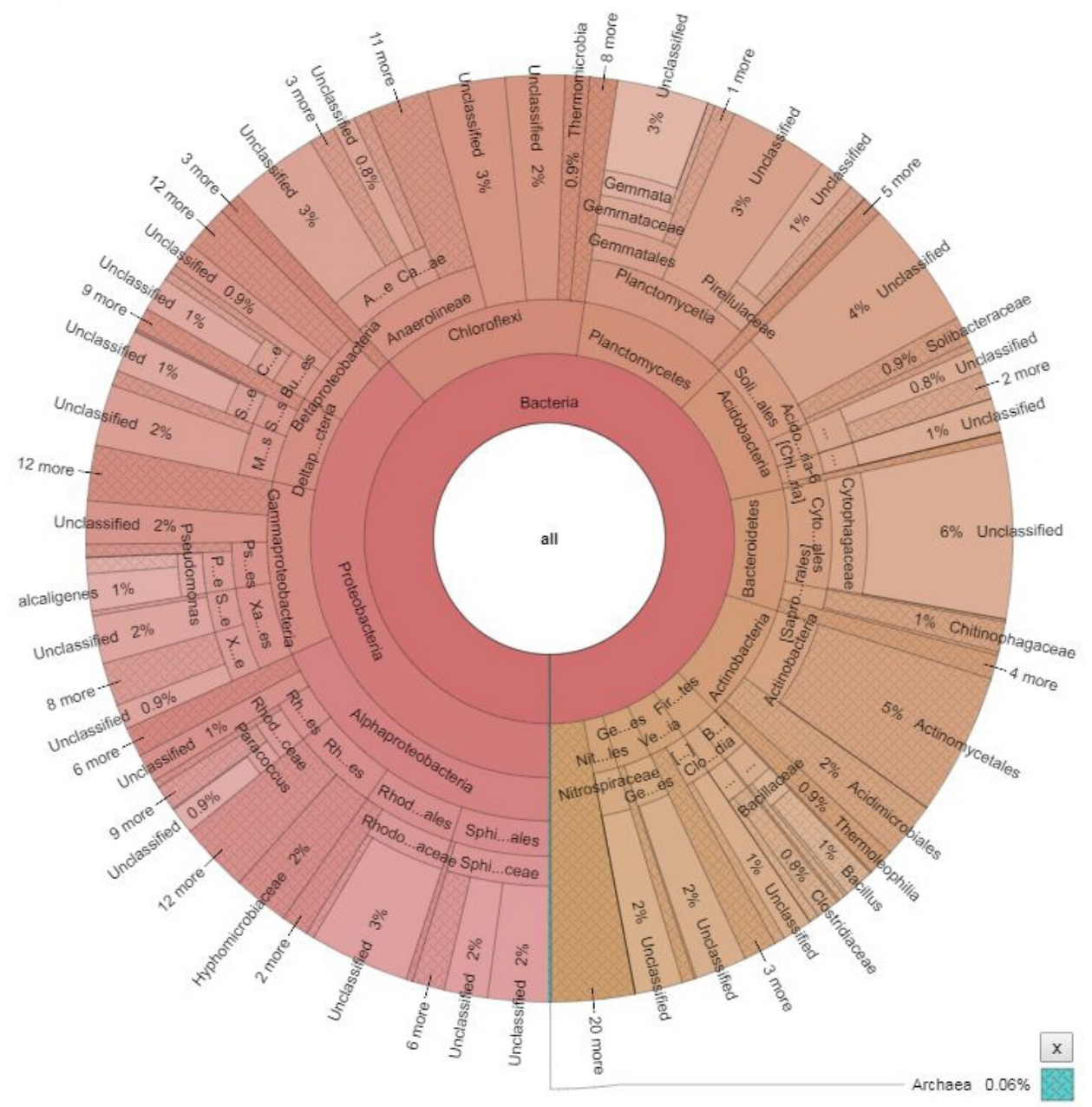

Figure 4.9. Whole community sequencing of bacterial population in CWS 


\subsection{Fungal community}

Though many reference data sets are available for fungi, internal transcribed spacer (ITS) data set is found to exhibit more diverse fungal community for evaluation from raw reads. Raw reads of 383,187 with total bases of $184,687,540$ based were read. ITS data was used for determination of fungal community in the representative sample of our study. The relative abundance of fungal phyla is presented in Fig. 4.10.

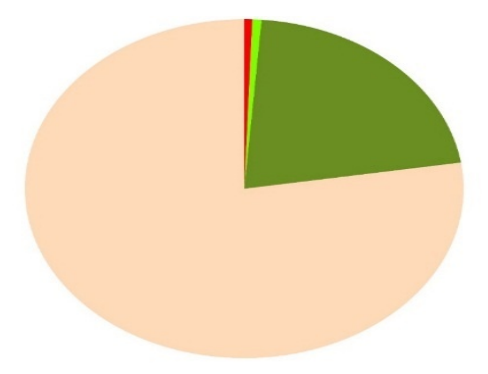

WCSITS Phylum legend

\begin{tabular}{|c|c|c|}
\hline Legends & Taxonomy & Abundance \\
\hline & k_Fungi,p_Ascomycota & $77.47 \%$ \\
\hline & k_Fungi;p_unidentified & $21.27 \%$ \\
\hline & k_Fungi,p_Zygomycota & $0.68 \%$ \\
\hline & k_Fungi;p_Basidiomycota & $0.58 \%$ \\
\hline
\end{tabular}

Figure 4.10. Relative abundance of fungal phyla

The ITS dataset identified only three phyla whereas, unidentified constitutes $21.27 \%$ of the total OTUs. The fungal community has the potential to remove phosphate, ammonia, TN and COD from domestic sewage and release extracellular enzymes for decomposition of plant litter. Fungi are more active and advantageous than bacteria in metabolising complex matter and has the capability to transform broad spectrum of compounds. Fungi are commercially used in industrial level and in WWTP for degradation of several contaminants. However, the applications of fungi in WWTP are still in infant stage though they act as powerful tool in wastewater treatment.

Ascomycota is reported as the dominant phyla in activated sludge WWTP. Ascomycota and Basidiomycota are the major classical decomposers and pollutant degraders in soil favouring organic, cellulose and lignin degradation contributing a vital role in carbon cycle. The beneficial effects of symbionts i.e. mychorrhizal association in heavy metal tolerant plants was reported [27]. Arbuscular mycorrhizae helps in chelating heavy metals beyond the rhizosphere by releasing some insoluble glycoproteins named glomalin. Mycorrhizal colonization in the heavy metal tolerant plants help in the uptake of heavy metals and plays a vital role in the protection of roots from heavy metal damage. Zinc crystalloids were deposited within the fungal mycelium and cortical cells of mycorrhizal roots of Euphorbia cyparissias grown in zinc contaminated wastes. The mycorrhizal fungal ecotypes in heavy metal contaminated sites are more tolerant to heavy metals than reference strains in uncontaminated soil.

Ascomycota (6.3-7.4\%) and Basidiomycota (1.5-1.7\%) were reported to be the most abundant phyla in 12 activated sludge samples from four WWTPs with stable removal efficiency of COD and TN for a longer run. The dominance of Ascomycota might have favoured the removal of COD and nitrogen from the system. Ascomycota absorbs nutrients from both live and dead bodies, with a significant rise upon increase in nitrogen and phosphorus. Ascomycota is an active player in removal of pharmaceutical pollutants like antibiotics [28]. Zygomycota metabolises xenobiotics. Cytochrome p450 super family of enzymes, present in Ascomycota, Basidiomycota and Zygomycota pertaining to novel degradation pathways [29]. 
The relative abundance of fungal classes are presented in Fig..4.11. At class level Eurotiomycetes predominates When nitric oxide (NO) was used to prevent biofouling in membrane bioreactor (MBR), there was a significant increase in Eurotiomycetes. Eurotiomycetes was dominant in the most contaminated (total petroleum hydrocarbon) sandy soil and the dominance range did not decrease upon increase in contamination. Eurotiomycetes was positively stimulated by fumigation. Many researchers concluded that Eurotiomycetes is capable of surviving in extreme conditions and produce extracellular and hydrolytic enzymes [30].

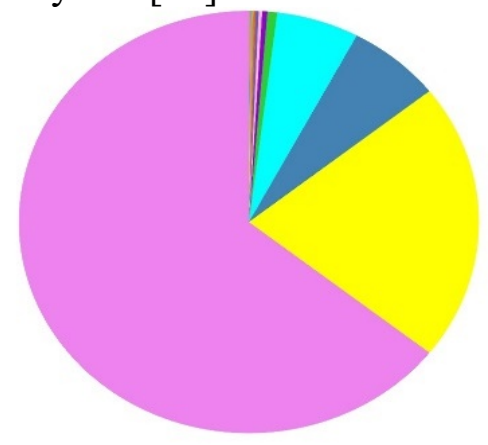

WCSITS Class legend

\begin{tabular}{|c|c|c|}
\hline Legends & Taxonomy & Abundance \\
\hline & k_Fungi;p_Ascomycota;c_Eurotiomycetes & $64.38 \%$ \\
\hline & k_Fungi;p_unidentified;c_unidentified & $21.27 \%$ \\
\hline & k_Fungi;p_Ascomycota;c_Sordariomycetes & $6.59 \%$ \\
\hline & k_Fungi;p_Ascomycota;c_Dothideomycetes & $5.8 \%$ \\
\hline & k_Fungi;p_Zygomycota;c_Incertae_sedis & $0.68 \%$ \\
\hline & k_Fungi;p_Basidiomycota;c_Tremellomycetes & $0.37 \%$ \\
\hline & k_Fungi;p_Ascomycota;c_unidentified & $0.24 \%$ \\
\hline & k_Fungi;p_Ascomycota;c_Incertae_sedis & $0.21 \%$ \\
\hline & k_Fungi;p_Basidiomycota;c_Agaricomycetes & $0.13 \%$ \\
\hline & k_Fungi;p_Ascomycota;c_Orbiliomycetes & $0.13 \%$ \\
\hline & k_Fungi;p_Ascomycota;c_Pezizomycetes & $0.08 \%$ \\
\hline & k_Fungi;p_Ascomycota;__Saccharomycetes & $0.04 \%$ \\
\hline k_Fungi;p_Basidiomycota;c_unidentified & $0.03 \%$ \\
\hline & k_Fungi;p_Basidiomycota;c_Microbotryomycetes & $0.03 \%$ \\
\hline & k_Fungi;p_Basidiomycota;c_Wallemiomycetes & $0.02 \%$ \\
\hline k_Fungi;p_Ascomycota;c_Leotiomycetes & $0.01 \%$ \\
\hline
\end{tabular}

Figure 4.11. Relative abundance of fungal class

Sordariomycetes was previously identified in industrial WWTP involved in degradation and removal of organics and xenobiotics [31]. Dothideomycetes is capable of digesting plant litter. Incertae sedis is indicated as one of the core microbial population existing in WWTP located at different geographic locations and its role in biological WWTP is not well explained. Tremellomycetes is reported to be present in flocular and granular sludge, it is unculturable and dominant in polar arctic circle bioreactors [32]. Agaricomycetes class is capable of degrading pharmaceutical active compounds and bioremediation of crude oil contaminated site [33]. Orbiliomycetes and Pezizomycetes are involved in the process of organic composting and cattle farm waste composting respectively. These classes might have decomposed the organic matter in CWS.

The members of the class Saccharomycetes are involved in bioremediation of heavy metals: cadmium, lead, chromium and manganese [34]. Microbotryomycetes regulates plant mineral nutrient cycling and resistant to heavy metals: iron, lead, manganese, cadmium, copper and zinc. Leotiomycetes has saphrophytic and parasitic relationship with wide range of plants. Eurotiomycetes and Leotiomycetes contain a large variety of dark septate endophytic enabling, efficient uptake of nutrients by plants. No published literature was available about Wallemiomycetes and its role in WWTP. 
The relative abundance of fungal orders are presented in Fig..4.12. At order level, Eurotiales predominates. Members of Eurotiales play a major role in global carbon cycle and nutrient uptake, excretes organic acids which solublises the unavailable potassium and makes it available for plant uptake. The unidentified order of the unidentified phyla represents $21.27 \%$ of the total OTUs.

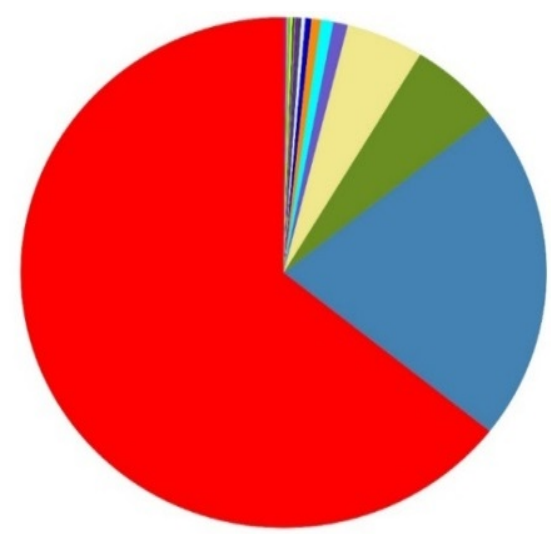

WCSITS Order legend

\begin{tabular}{|c|c|c|}
\hline Legends & Taxonomy & Abundance \\
\hline & k_Fungi;p_Ascomycota;c_Eurotiomycetes;0_Eurotiales & $64.34 \%$ \\
\hline & k_Fungi;p_unidentified;c_unidentified;0_unidentified & $21.27 \%$ \\
\hline & k_Fungi;p_Ascomycota;c_Sordariomycetes;0_Hypocreales & $5.7 \%$ \\
\hline & k_Fungi;p_Ascomycota;c_Dothideomycetes;0_Pleosporales & $4.77 \%$ \\
\hline & k_Fungi;p_Ascomycota;c_Dothideomycetes;0_Capnodiales & $0.89 \%$ \\
\hline & k_Fungi;p_Ascomycota;c_Sordariomycetes;0_Sordariales & $0.77 \%$ \\
\hline & k_Fungi;p_Zygomycota;c_Incertae_sedis;0_Mucorales & $0.58 \%$ \\
\hline & k_Fungi;p_Basidiomycota;c_Tremellomycetes;0_Tremellales & $0.37 \%$ \\
\hline & k_Fungi;p_Ascomycota;c_unidentified;o_unidentified & $0.24 \%$ \\
\hline & k_Fungi;p_Ascomycota;c_Incertae_sedis;0_Incertae_sedis & $0.21 \%$ \\
\hline & k_Fungi;p_Ascomycota;c_Orbiliomycetes;0_Orbiliales & $0.13 \%$ \\
\hline & k_Fungi;p_Ascomycota;c_Sordariomycetes;0_Microascales & $0.1 \%$ \\
\hline & k_Fungi;p_Zygomycota;c_Incertae_sedis;0_Kickxellales & $0.1 \%$ \\
\hline & k_Fungi;p_Basidiomycota;c_Agaricomycetes;0_Agaricales & $0.1 \%$ \\
\hline & k_Fungi;p_Ascomycota;c_Dothideomycetes;0_Incertae_sedis & $0.09 \%$ \\
\hline & k_Fungi;p_Ascomycota;c_Pezizomycetes;0_Pezizales & $0.08 \%$ \\
\hline & k_Fungi;p_Ascomycota;c_Dothideomycetes;0_Botryosphaeriales & $0.04 \%$ \\
\hline & k_Fungi;p_Ascomycota;c_Saccharomycetes;0_Saccharomycetales & $0.04 \%$ \\
\hline & k_Fungi;p_Basidiomycota;c_unidentified;0_unidentified & $0.03 \%$ \\
\hline & Others & $0.16 \%$ \\
\hline
\end{tabular}

Figure 4.12. Relative abundance of fungal order

At the maturing stage of compost, increased concentration of Eurotiales (32.5\%) Hypocreales $(25.4 \%)$ and Microascales (20.2\%) were reported [35]. Hypocreales presence is reported in bioaerosols of coal mine. Pleosporales is reported to be present in textile WWTP. Pleosporales and Capnodiales in WWTP take part in glycolysis and esterification. Sordariales, Orbiliales and Mucorales are dominant in bioorganic fertilizers. Tremellales and Agaricales possess genes which play a key role in degradation of recalcitrant organic matter [36]. Insertae sedis is reported to exist in WWTP and activated sludge and exhibits a wide range of metabolic activities including nitrogen fixation and metal oxidation. Kickxellales are saprobes which participate in organic degradation. Pezizales significantly increase at increasing $\mathrm{pH}$. Botrysphaeriales and Saccharomycetales significance in WWTP is not explored yet. 
The relative abundance of fungal family is presented in Fig. 4.13. At family level, Trichosporonaceae predominates. Trichosporon in Trichosporonaceae family dominated in fungi domain in bioreactor playing an important role in denitrification and Oxidation of ammonia [32]. The family Nectriaceae has a cosmopolitan distribution and enriched upon organic amendments in wheat field, indicating its potential to degrade organics. Members of Incertea sedis, Pleosporaceae and Cunninghamellaceae family are reported to be present in soil contaminated with heavy metals: zinc, lead, cadmium, copper iron and manganese [37]. Few members of the family Mycosphaerellaceae are reported to exhibit anti-parasitic and cytotoxic activity. Orbiliaceae produces nematode trap in the presence of urea in the system [38] and might have trapped the nematodes in CWS as sewage contains urea. Members belonging to Microascaceae are capable of treating hydrocarbons. Members of Coprinaceae were capable of producing laccases and peroxidases capable of degrading lignin. The metabolic activity of families Trichosporanaceae, Kickxellaceae, Erimomycetaceae and Hypocreaceae in WWTP were not reported in any published literature.

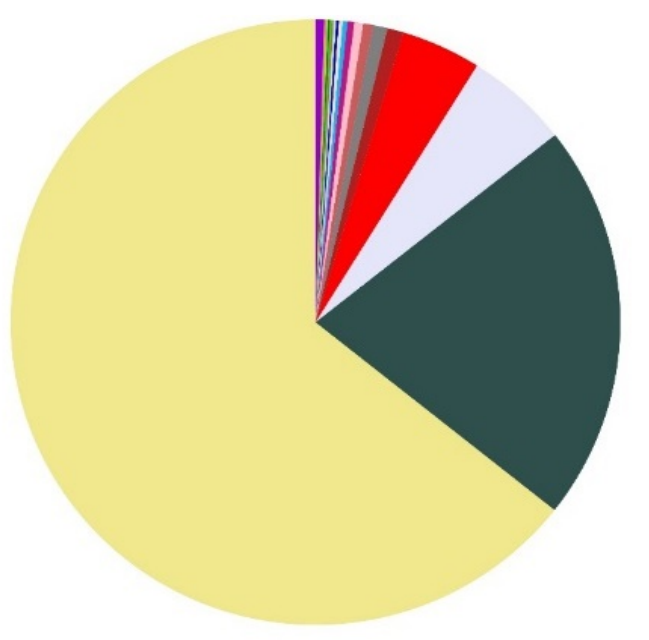

WCSITS Family legend

\begin{tabular}{|c|c|c|}
\hline Legends & Taxonomy & Abundance \\
\hline & k_Fungi;p_Ascomycota;c_Eurotiomycetes;0_Eurotiales;f_Trichocomaceae & $64.34 \%$ \\
\hline & k_Fungi;p_unidentified;c_unidentified;o_unidentified;f_unidentified & $21.27 \%$ \\
\hline & k_Fungi;p_Ascomycota;c_Sordariomycetes;0_Hypocreales;f_Nectriaceae & $5.49 \%$ \\
\hline & k_Fungi;p_Ascomycota;c_Dothideomycetes;0_Pleosporales;f_Incertae_sedis & $4.23 \%$ \\
\hline & k_Fungi;p_Ascomycota;'c_Dothideomycetes;0_Capnodiales;f_Mycosphaerellaceae & $0.86 \%$ \\
\hline & k_Fungi;p_Ascomycota;___Sordariomycetes;0_Sordariales;f_Chaetomiaceae & $0.77 \%$ \\
\hline & k_Fungi;p_Zygomycota;c_Incertae_sedis;o_Mucorales;f_Cunninghamellaceae & $0.52 \%$ \\
\hline & k_Fungi,p_Ascomycota;c_Dothideomycetes;0_Pleosporales;f_Pleosporaceae & $0.47 \%$ \\
\hline & k_Fungi;p_Basidiomycota;c_Tremellomycetes;0_Tremellales;f_Trichosporonaceae & $0.37 \%$ \\
\hline & k_Fungi;p_Ascomycota;'c_unidentified;o_unidentified;f_unidentified & $0.24 \%$ \\
\hline & k_Fungi;p_Ascomycota;c_Incertae_sedis;0_Incertae_sedis;f_Incertae_sedis & $0.21 \%$ \\
\hline & k_Fungi,p_Ascomycota;___Sordariomycetes;0_Hypocreales;f_Incertae_sedis & $0.14 \%$ \\
\hline & k_Fungi;p_Ascomycota;c_Orbiliomycetes;0_Orbiliales;__Orbiliaceae & $0.13 \%$ \\
\hline & k_Fungi;p_Ascomycota;c_Sordariomycetes;0_Microascales;f_Microascaceae & $0.1 \%$ \\
\hline & k_Fungi;p_Zygomycota;___ncertae_sedis;,_Kickxellales;f_Kickxellaceae & $0.1 \%$ \\
\hline & k_Fungi;p_Ascomycota;c_Dothideomycetes;0_Incertae_sedis;f_Eremomycetaceae & $0.09 \%$ \\
\hline & k_Fungi;p_Ascomycota;c_Pezizomycetes;0_Pezizales;f_Incertae_sedis & $0.08 \%$ \\
\hline & k_Fungi;p_Basidiomycota;c_Agaricomycetes;0_Agaricales;,_Coprinaceae & $0.07 \%$ \\
\hline & k_Fungi;p_Ascomycota;c_Sordariomycetes;0_Hypocreales;__Hypocreaceae & $0.06 \%$ \\
\hline & Others & $0.45 \%$ \\
\hline
\end{tabular}

Figure 4.13. Relative abundance of fungal family 
The relative abundance of fungal genera are presented in Fig. 4.14. At the genus level, Penicillium predominates

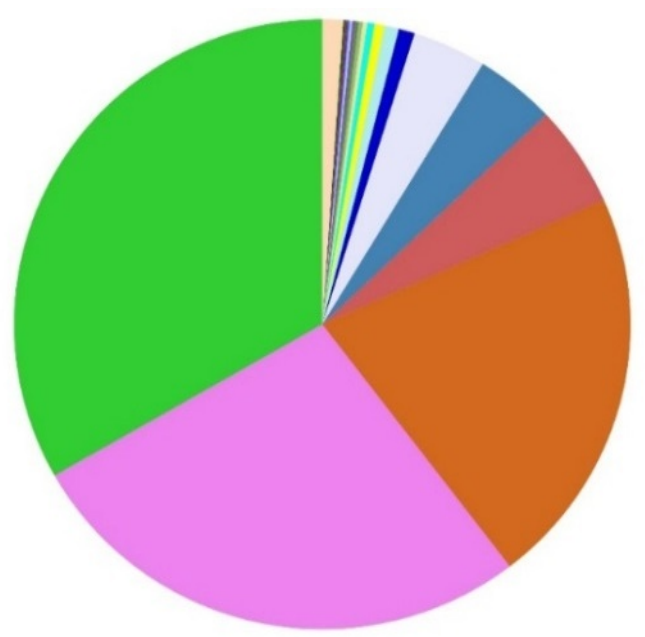

WCSITS Genus legend

\begin{tabular}{|c|c|c|}
\hline Legends & Taxonomy & Abundance \\
\hline & k_Fungi;p_Ascomycota;c_Eurotiomycetes;0_Eurotiales;f_Trichocomaceae;g_Penicillium & $33.26 \%$ \\
\hline & k_Fungi,p_Ascomycota;_c_Eurotiomycetes;0_Eurotiales;;_Trichocomaceae;g_Paecilomyces & $27.15 \%$ \\
\hline & k_Fungi;ip_unidentified;c_unidentified;0_unidentified;f_unidentified;g_unidentified & $21.27 \%$ \\
\hline & k_Fungi;p_Ascomycota;c_Sordariomycetes;0_Hypocreales;f_Nectriaceae;g_Haematonectria & $5.39 \%$ \\
\hline & k_Fungi'p_Ascomycota,c_Dothideomycetes;o_Pleosporales;f_Incertae_sedis,g_Phoma & $4.22 \%$ \\
\hline & k_Fungi;p_Ascomycota;c_Eurotiomycetes;0_Eurotiales;f_Trichocomaceae;g_Aspergillus & $3.87 \%$ \\
\hline & k_Fungi,p_Ascomycota;'c_Dothideomycetes;'0_Capnodiales;f_Mycosphaerellaceae'g_Cladosporium & $0.84 \%$ \\
\hline & k_Fungi;p_Ascomycota;c_Sordariomycetes;0_Sordariales,f_Chaetomiaceae;g_Humicola & $0.77 \%$ \\
\hline & k_Fungi;p_Zygomycota;c_Incertae_sedis;o_Mucorales;f_Cunninghamellaceae;g_Cunninghamella & $0.52 \%$ \\
\hline & k_Fungi,p_Basidiomycota;__Tremellomycetes;0_Tremellales;i_Trichosporonaceae;g_Trichosporon & $0.37 \%$ \\
\hline & k_Fungi;p_Ascomycota;c_unidentified;0_unidentified;f_unidentified;g_unidentified & $0.24 \%$ \\
\hline & k_Fungi,p_Ascomycota;'c_Dothideomycetes;;_Pleosporales;f_Pleosporaceae;g_Cochliobolus & $0.21 \%$ \\
\hline & k_Fungi,p_Ascomycota;c_Incertae_sedis;0_Incertae_sedis;ifIncertae_sedis;g_Dokmaia & $0.17 \%$ \\
\hline & k_Fungi;p_Ascomycota;c_Dothideomycetes;0_Pleosporales;f_Pleosporaceae;g_Setosphaeria & $0.17 \%$ \\
\hline & k_Fungi,p_Zygomycota;__Incertae_sedis;o_Kickxellales;,_Kickxellaceae;g_Coemansia & $0.1 \%$ \\
\hline & k_Fungi;__Ascomycota;c_Dothideomycetes;0_Incertae_sedis;f_Eremomycetaceae;g_Arthrographis & $0.09 \%$ \\
\hline & k_Fungi;i_Ascomycota;_c_Orbiliomycetes;0_Orbiliales;f_Orbiliaceae;g_Drechslerella & $0.09 \%$ \\
\hline & k_Fungi;p_Ascomycota;C_Sordariomycetes;0_Hypocreales;f_Nectriaceae;g_Fusarium & $0.09 \%$ \\
\hline & k_Fungip_Ascomycota;'c_Sordariomycetes;0_Hypocreales;f_Incertae_sedis;g_Cephalosporium & $0.09 \%$ \\
\hline & Others & $1.09 \%$ \\
\hline
\end{tabular}

Figure 4.14. Relative abundance of fungal genera

The genera Penicillium, Fusarium and Aspergillus are reported to survive in acid mine drainage, industrial wastewater and are reported as multi metal $(\mathrm{Cd}, \mathrm{Cr}$ and $\mathrm{Ni})$ resistant [39]. Paecilomyces is used as biocatalyst in industries for production of enzymes and detoxifying agent in treating industrial residue [40]. Paecilomyces and Aspergillus are reported to be cadmium tolerant and capable of bioaccumulation. They were isolated from soil contaminated with tannery wastewater and utilized in bioremediation of chromium [41]. Aspergillus and Penicillium solublises inorganic and organic phosphate in liquid and soil medium and might have helped in the removal of phosphate from wastewater. 
Some of the species of Haematonectria are capable of decolourising textile dyes. In Phoma salt tolerant genes were reported and isolated from root nodules of leguminous plant involved in nitrogen fixation [42]. Phoma is a hyperaccumulator of cadmium. Cladosporium genus is capable of removing textile dyes and pollutants released from textile industry [43]. Most of the members of the genera Fusarium, Aspergillus and Humicola are resistant to heavy metals. Aspergillus and Humicola actively participate in composting. Cunninghamella, Aspergillus and Penicillium are heavy metal tolerant. Cunninghamella is potential in accumulating phosphate in the form of polyphosphate and degrading nitrogen in the form of ammonium and nitrate. Hence, can be used in biotechnological processes for nutrient removal. This genus might have generously contributed for the removal of nutrients. Trichosporon is considered as one of the genera contributing to purification of wastewater by denitrification [44]. The antioxidant system against oxidative stress induced by heavy metals $\mathrm{Cr}$ and Cd were reported in Trichosporon to survive in conditions of heavy metal stress. Cochliobolus is isolated from gold and gemstone mining sites, mercury-contaminated site and is stated to be heavy metal tolerant [45]. Dokmaia, Setosphaeria, Coemansia, Drechslerella and Arthrographis produces enzyme laccase in treating textile wastewater and certain xenobiotics. Fusarium is present in sewage and part of the biofilm formation. Fusarium was isolated from root nodules of leguminous plant involved in nitrogen fixation [42].

The relative abundance of fungal species is presented in Fig. 4.15. At species level, Penicillium HF12085 predominates, however, its role and presence in wastewater treatment is not available in published literature and needs exploration. Paecilomyces lilacinus and Aspergillus niger are capable of tolerating multi metal: $\mathrm{Cr}, \mathrm{Ni}, \mathrm{Fe}, \mathrm{Mn}, \mathrm{Cd}$ and $\mathrm{Pu}$.

Paecilomyces lilacinus and Aspergillus niger are capable of tolerating multi metal: $\mathrm{Cr}, \mathrm{Ni}, \mathrm{Fe}$, $\mathrm{Mn}, \mathrm{Cd}$ and Pu. Paecilomyces lilacinus is used in treatment of tannery wastewater [41]. A. flavus, . niveus and $A$. terreus are capable of removing almost all the heavy metals existing in industrial wastewaters Fusarium solani uptakes heavy metals lead, zinc, chromium, cobalt, nickel and copper. Aspergillus niger is capable of removing $72-90 \%$ of COD, $65.4 \%$ of TN and $75-100 \%$ removal of multi metals: uranium, mercuric chloride, lead, cadmium, copper and utilised in treating textile wastewater [46].

Enhanced sulphate removal from wastewater using Aspergillus niger and Aspergillus flavus was investigated and proved. Aspergillus niger, Fusarium sp. and Aspergillus flavus were potential in removal of nitrogen and phosphate from wastewater [47]. Penicillium janthinellum is capable of reducing aluminium metal toxicity and was immobilised on substrates used for treatment of paper mill effluent [48]. Aspergillus terreus immobilised on various substrates were used for the reduction of TS, alkalinity, EC, nitrate, nitrite, BOD, COD and colour removal from textile effluents [49]. 


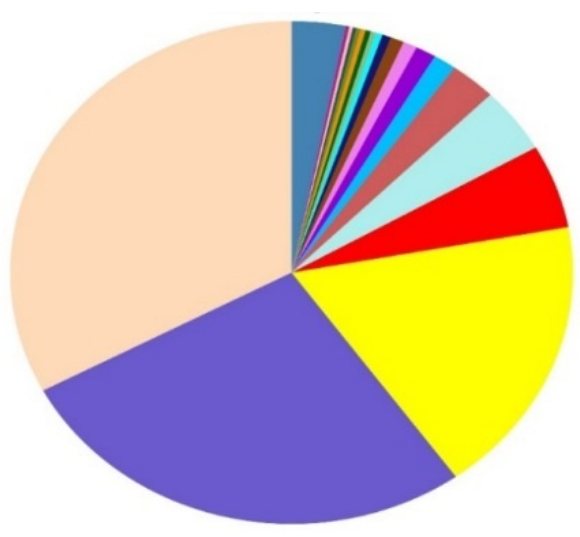

WCSITS Species legend

\begin{tabular}{|c|c|c|}
\hline Legends & Taxonomy & Abundance \\
\hline & k_Fungi;P_Ascomycota;c_Eurotiomycetes;0_Eurotiales;f_Trichocomaceae;g_Penicillium;s_Penicillium_sp_HF12085 & $32.77 \%$ \\
\hline & k_Fungi;p_Ascomycota;c_Eurotiomycetes;0_Eurotiales;__Trichocomaceae;g_Paecilomyces;s_Paecilomyces_lilacinus & $27.15 \%$ \\
\hline & 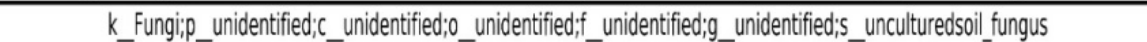 & $17.97 \%$ \\
\hline & k_Fungi;p_Ascomycota;c_Sordariomycetes;0_Hypocreales;__Nectriaceae;'g_Haematonectria;s_Fusarium_solani & $5.39 \%$ \\
\hline & k_Fungi;p_Ascomycota;___Dothideomycetes;0_Pleosporales;'f_Incertae_sedis;'g_Phoma;'s_Phoma_tropica & $4.22 \%$ \\
\hline & k_Fungilip_unidentified;c__unidentified; $0 \_$unidentified;f_unidentified; g_unidentified;s_unculturedfungus & $2.66 \%$ \\
\hline & k_Fungi;p_Ascomycota;c_Eurotiomycetes;0_Eurotiales;f_Trichocomaceae;g_Aspergillus;s_Aspergillus_niger & $1.32 \%$ \\
\hline & k_Fungi;p_Ascomycota;c_Eurotiomycetes;0_Eurotiales;f_Trichocomaceae;g_Aspergillus;s_Aspergillus_SP_ASR_215 & $1.18 \%$ \\
\hline & k_Fungi,p_Ascomycota;___Dothideomycetes;0_Capnodiales;;_Mycosphaerellaceae,g_Cladosporium;s_Cladosporium_sp_F128 & $0.81 \%$ \\
\hline & k_Fungi;p_Ascomycota;c_Sordariomycetes;0_Sordariales;f_Chaetomiaceae;g_Humicola;s_Humicola_sp_L_2 & $0.77 \%$ \\
\hline & k_Fungi;p_Zygomycota;c_Incertae_sedis;0_Mucorales;__Cunninghamellaceae;g_Cunninghamella;s_Cunninghamella_blakesleeana & $0.52 \%$ \\
\hline & k_Fungi';__unidentified;'__unidentified;0_unidentified;'_unidentified;g_unidentified;s_fungal_endophyte_sp_P1628A & $0.37 \%$ \\
\hline & k_Fungi;p_Ascomycota;__EEurotiomycetes;0_Eurotiales;__Trichocomaceae;g_Aspergillus;s_Aspergillus_flavus & $0.31 \%$ \\
\hline & k_Fungi;p_Ascomycota;__EEurotiomycetes;0_Eurotiales;f_Trichocomaceae;'g_Aspergillus;'s_Aspergillus_niveus & $0.31 \%$ \\
\hline & k_Fungi;p_Basidiomycota;___Tremellomycetes;0_Tremellales;__Trichosporonaceae;g_Trichosporon;s_Trichosporon_scarabaeorum & $0.29 \%$ \\
\hline & k_Fungi;p_Ascomycota;c_Eurotiomycetes;0_Eurotiales;f_Trichocomaceae;g_Aspergillus;s_Aspergillus_sp_HF12437 & $0.24 \%$ \\
\hline & k_Fungi;p_Ascomycota;c__Dothideomycetes;0_Pleosporales;f_Pleosporaceae;g_Cochliobolus;s_Cochliobolus_lunatus & $0.21 \%$ \\
\hline & k_Fungi,p_Ascomycota;_c_Eurotiomycetes;0_Eurotiales;;_Trichocomaceae;'g_Penicillium;s_Penicillium janthinellum & $0.21 \%$ \\
\hline & k_Fungi;p_Ascomycota;__EEurotiomycetes;0_Eurotiales;__Trichocomaceae';g_Aspergillus;s_Aspergillus_terreus & $0.19 \%$ \\
\hline & Others & $3.1 \%$ \\
\hline
\end{tabular}

Figure 4.15. Relative abundance of fungal species

Aspergillus ASR 215, Cladosporium F128, Humicola L2, fungal endophyte P1628, Trichosporon scarabaeorum, Aspergillus HF 12437 and Cochliobolus lunatus were not reported in WWTP and its role in wastewater treatment is not explored yet.

Total of 165 OTUs were observed with a shanon alpha diversity of 3.432 . The species richness can be calculated from rarefaction curve. Diversity and rarefaction measure of observed OTUs of the bacterial genome was calculated using $\alpha$ diversity metrics is presented in Fig. 4.16. 


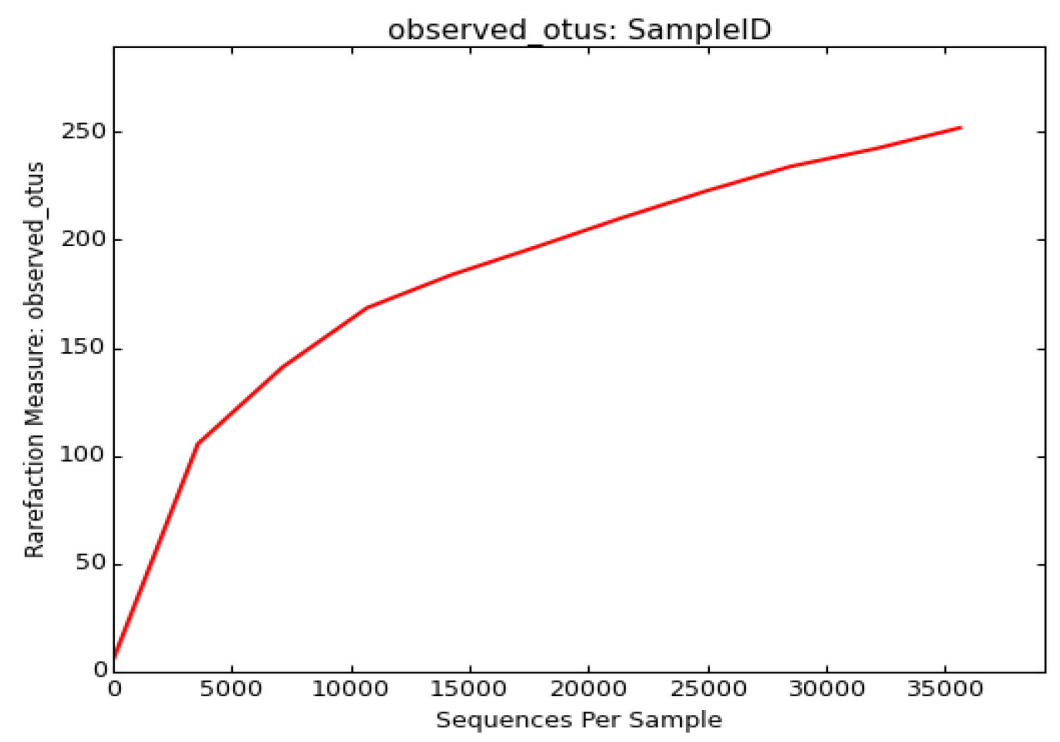

Figure 4.16. Rarefaction measure of observed OTUs in fungal genome

Steep slope on the left indicates that a large fraction of the species remains to be discovered. The vertical axis displays the diversity of the community and horizontal displays the number of sequences considered in the diversity calculation. The whole community sequencing of fungal population is presented in Fig. 4.17 using Krona software.

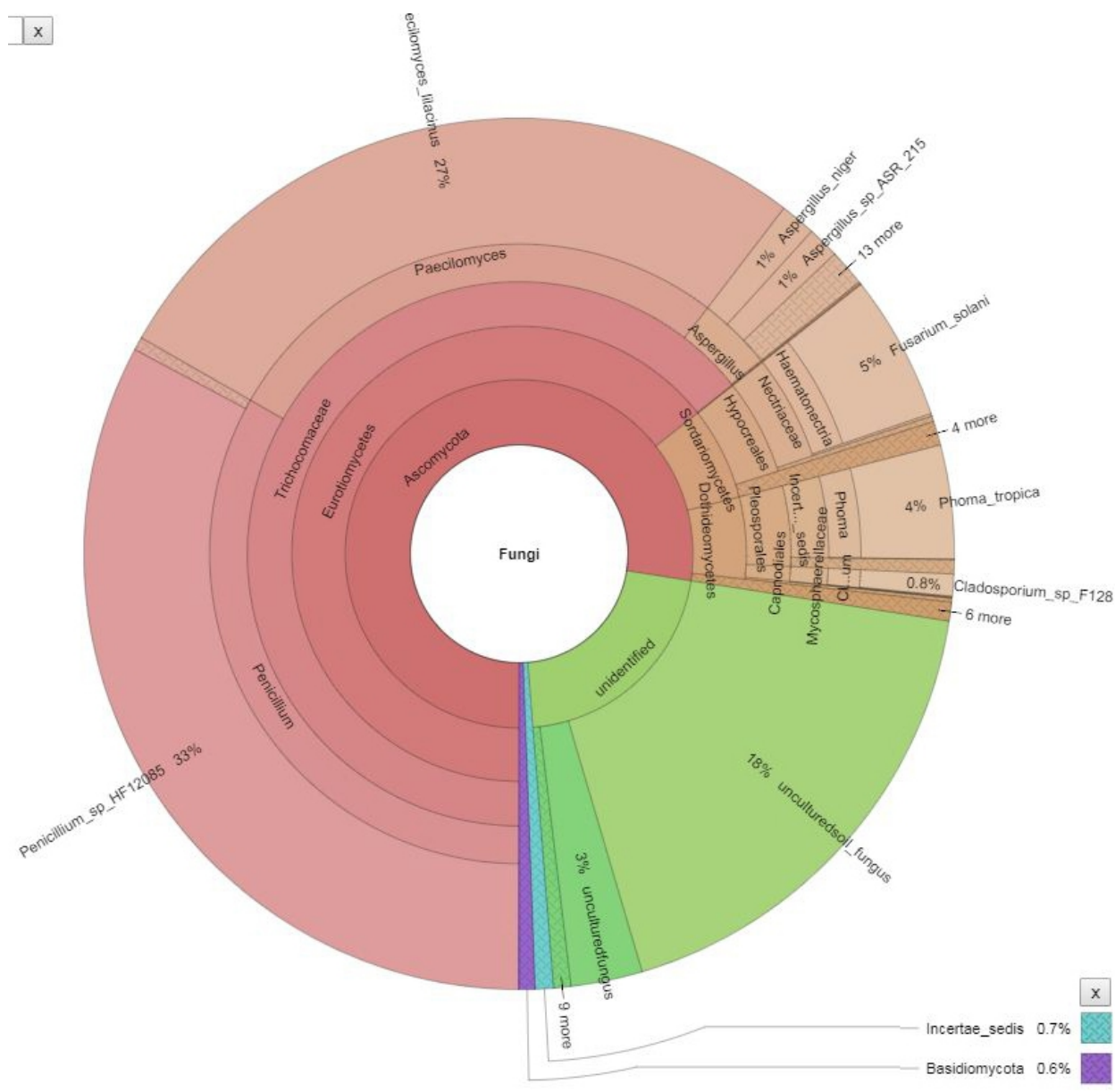

Figure 4.17. Whole community sequencing of fungal population in CWS 


\section{Conclusion}

Microbes in wetland play a pivotal role in the degradation and removal of organics, nutrients and pollutants from wastewater during the treatment process. From the study, the list of microbes in CWS was identified. However, at species level, it remained majorly unclassified in bacterial kingdom. Some of the unculturable bacterial species were also identified. The role of fungi in wastewater treatment need exploration. The identification of specific phyla in an environment helps us to get an idea of the degradation activity in that particular environment. The role of microbes in cleaning up the environment to sustain ecological balance is undeniable. A spectacular improvement in the database is recommended for identification of all the species involved.

\section{Conflict of interest}

The author declares no conflict of interest.

\section{Acknowledgement}

The author wishes to thank the University Grants Commission, New Delhi for supporting her PhD studies.

\section{References}

[1] U. Stottmeister et al., Constructed wetlands and their performance for treatment of water contaminated with arsenic and heavy metals, Soil and Water Pollution monitoring protection and remediation, Springer, Dordrecht, 2006.

[2] A.F. Desta et al., Microbial community structure and diversity in an integrated system of anaerobic-aerobic reactors and a constructed wetland for the treatment of tannery wastewater in Modjo Ethiopia, Plos one. 9 (2014) e115576.

[3] I.N. Balcom et al., Metagenomic analysis of an ecological wastewater treatment plants microbial communities and their potential to metabolize pharmaceuticals, F1000research. 5 (2016) 1-26.

[4] A. Mark Ibekwe, C.M. Grieve, S.R. Lyon, Characterisation of microbial communities and composition in constructed dairy wetland wastewater effluent, Applied Environmental Microbiology. 69 (2003) 95060-95069.

[5] P. Dudhagara et al., Web resources for metagenomic studiesGenomics, Proteomics and Bioinformatics. 13 (2015) 296-303.

[6] A. Cydzik-Kwiatkowska, M. Zielinska, Bacterial communities in full-scale wastewater treatment systems, World Journal of Microbiology and Biotechnology. 32 (2016) 1-8.

[7] W. Guan et al., Influence of substrate type on microbial community structure in vertical flow constructed wetland treating polluted river water, Environmental Science Pollution and Research. 22 (2015) 16202-16209.

[8] P. Arroyo et al., Comparative analysis of the composition of bacterial communities from two constructed wetlands for municipal and swine wastewater treatment, Journal of Water Health. 8 (2010) 147-157.

[9] S. Takaich et al., Carotenoids of Gemmatimonas aurantiaca (Gemmatimonadetes): identification of a novel carotenoid, deoxyoscillol 2- rhamnoside, and proposed biosynthetic pathway of oscillol 2,2'dirhamnoside, Microbiology. 156 (2009) 757-763.

[10] H. Sun et al., Myriophyllum aquaticum constructed wetland effectively removes nitrogen in swine wastewater, Frontiers in Microbiology. 8 (2017) 1-14.

[11] DR. Speth et al., Genome based microbial ecology of anammox granules in a full-scale wastewater treatment system, Nature communications. 7 (2016) $11172-11116$ 
[12] Q. Zhou, T. Chen, S. Han, Characteristics of bacterial communities in Cyanobacteriablopoming aquaculture wastewater influenced by the phytoremediation with water hyacinth, Water. 9 (2017) 1-11.

[13] O.V. Tsoy et al., Nitrogen fixation and molecular oxygen:Comparative genomic reconstruction of transcription regulation in alphaproteobacteria, Frontiers in Microbiology. 7 (2016) 1343

[14] H. Yin et al., An integrated insight into the response of sedimentary microbial communities to heavy metal contamination, Scientific Reports. 5 (2015) 14266.

[15] P. Swiatczak, A. Cydzik- Kwiatkowska, P. Rusanowska, Microbiota of anaerobic digestors in a full scale wastewater treatment plant, Archives of Environmental Protection. 43 (2018) 5360.

[16] A.M. Kielak et al., The ecology of Acidobacteria: moving beyond genes and genomes, Frontiers in Microbiology. 7 (2016) 744.

[17] H. Tian et al., Process performance and bacterial community structure under increasing influent disturbances in a membrane aerated biofilm reactors, Journal of Microbiology and Biotechnology. 26 (2016) 373-384.

[18] J.M. Hultman et al., Host range of antibiotic resistance genes in wastewater treatment plant influent and effluent, FEMS Microbiology Ecology. 94 (2018) 1-10.

[19] L. Drewniak et al., Physiological and metagenomic analyses of microbial mats involved in self -purification of mine waters contaminated with heavy metals, Frontiers in Microbiology. 7 (2016) 1252.

[20] B. Zhang et al., Seasonal bacterial community succession in four typical wastewater treatment plants: correlations between core microbes and process performance, Scientific Reports. $8(1)$ (2018) 4566.

[21] D.D. Meyer et al., Bacterial communities involved in sulfur transformations in wastewater treatment plants, Applied Microbiology and Biotechnology. 100 (2016) 10125-10135.

[22] S.J. Mcllroy et al., Genomic and insitu investigations of the novel uncultured Chlorflexi associated with 0092 morphotype filamentous bulking in activated sludge, ISME Journal. 10 (2016) 2223-2234.

[23] S. Zielinska et al., First insight into microbial community composition in a phosphogypsum waste heap soil, Acta Biochimica Polonica. 64 (2017) 1-6.

[24] M.H. Gerardi, Wastewater bacteria, Wiley Interscience, New Jersy, 2006.

[25] Q. Ma et al., Identification of the microbial community composition and structure of coal-mine wastewater treatment plants, Microbiology Research. 175 (2015) 1-5.

[26] T.A. Vishnivetskaya et al., Mercury and other heavy metals influence bacterial community structure in contaminated Tennessee streams, Applied and Environmental Microbiology. 77 (2011) 302-311.

[27] M. Miransari, Arbuscular mycorrhizal fungi and heavy metal tolerance in plants in: Q.S. Wu (Ed.), Arbuscular Mycorrhizas and stress tolerance of plants, Springer, Singapore, 2017, pp. 2659-2668.

[28] M. Naushad Life cycle assessment of wastewater treatment, CRC press, BocaRaton, Florida, 2018.

[29] D.R. Olicon- Hernandez, J. Gonzalez- Lopez, E. Aranda, Overview on the biochemical potential of filamentous fungi to degrade pharmaceutical compounds, Frontiers in Microbiology. 8 (2017) 1792.

[30] L. Chen et al., Responses of soil microeukaryotic communities to short-term fumigationincubation revealed by MiSeq amplicon sequencing, Frontiers in Microbiology. 6 (2015) 1149. 
[31] L.M. Silva- Bedoya et al., Bacterial community analysis of an industrial wastewater treatment plant in Colombia with screening for lipid-degrading microorganisms, Microbiology Research. 192 (2016) 313-325.

[32] A. Gonzalez-Martinez et al., Microbial ecology of full-scale wastewater treatment systems in the polar Arctic circle: Archaea, bacteria and fungi, Scientific Reports. 8 (2018) 2208.

[33] M.M. Mohammadi-Sichani et al., Bioremediation of soil contaminated crude oil by Agaricomycetes, Journal of Environmental Health Science and Engineering. 15 (2017) 1-6.

[34] M. Fadel et al., Biosorption of manganese from groundwater by biomass of Saccharomyces cerevisiae, HBRC Journal. 13 (2017) 106-113.

[35] A. Langarica-Fuentes et al., Fungal succession in an in-vessel composting system characterized using 454 pyrosequencing, FEMS Microbiology. Ecology. 88(2) (2014) 296-308.

[36] E.E. Kuramae et al., Structural and functional variation in soil fungal communities associated with litter bags containing maize leaf, FEMS Microbiology. Ecology. 84 (2013) 519-531.

[37] AM. Abdel-Azeem et al., Occurrence and diversity of microbiota in heavy metal contaminated sediments of Mediterranean coastal lagoon El-Manzala, Egypt, Mycosphere. 6 (2015) 228-240.

[38] X. Wang, G. Li, C.G. Zou Bacteria can mobilize nematode-trapping fungi to kill nematodes. Nature Communications. 5 (2014) 1-9.

[39] S. Das, H. Ranjan, Dash Handbook of metal-microbe interactions and bioremediation, CRC press, Bocaraton, Florida, 2017.

[40] VS. Ferreira-Leitao et al., The protagonism of biocatalysis in green chemistry and its environmental benefits, Catalysts. 7 (2017) 1-34.

[41] S. Sharma, P. Malaviya, Bioremediation of tannery wastewater by chromium resistant novel fungal consortium, Ecological Engineering. 91 (2016) 419-425.

[42] K. Widin, B.W. Kennedy, Effect of chemical soil treatment on plant growth, nitrogen fixation, and fungal colonization of Rhizobium nodules of Soybeans', Ecology and Epidemiology. 73 (1983) 429- 430.

[43] V.D. Jakovljevic, M.M. Vrvic, Potential of pure and mixed cultures of Cladosporium cladosporioides and Geotrichum candidum for application in bioremediation and detergent industry', Saudi Journal of Biological Sciences. 25 (2018) 529-536.

[44] A. Biedunkiewicz, T. Ozimek, Qualitative and quantitative changes of potentially pathogenic fungi Cunninghamella elegans in a hydrophyte wastewater treatment plant, Polish Journal of Environmental Studies. 18 (2008) 161-166.

[45] OG. Oladipo et al., Heavy metal torerance traits of filamentous fungi isolated from gold and gemstone mining sites, Brazilian Journal of Microbiology. 4 (2018) 29-37.

[46] A. Bahobil et al., Fungal biopsorption for cadmium and mercury heavy metal ions isolated from some polluted localities in KSA, International Journal of Current Microbiology and Applied Sciences. 6 (2017) 2138-2154.

[47] T.A. Adelani-Akande, O.B. Akpor, B.I. Aderiya, Investigation of the role of selected fungal strains in the removal of phosphate and nitrate in synthetic wastewater' Annual Research \& Review in Biology. 4 (2014) 1045-1058.

[48] R. Prasad, Mycoremediation and environmental sustainability: Fungal biology, Springer, Switzerland, 2018.

[49] V.G. Jogdand et al., Remediation of textile industry wastewater using immobilized Aspergillus terreus, European Journal of Experimental Biology. 2 (2012) 1550-1555. 